\title{
Club Virtual: estrategia de enseñanza y aprendizaje para el fortalecimiento de la lectura crítica
}

\author{
Ángela María Bonilla Vergara 1들 \\ Andrea Carolina Triana Guerra ${ }^{1}$ (D) \\ Alexandra María Silva Monsalve ${ }^{1}$ (D) \\ ${ }^{1}$ Universidad de Santander (UDES), Colombia
}

Resumen. La implementación de un club virtual de lectura como estrategia de enseñanza y aprendizaje surge con el propósito de fortalecer la lectura crítica. En este sentido, se fundamentó el marco teórico y conceptual para sustentar epistemológicamente la propuesta. Metodológicamente se plantea la investigación bajo el método cualitativo con diseño investigación-acción, en este sentido se diseña una propuesta de cambio para la comunidad educativa. La población estuvo conformada por cuarenta (40) estudiantes de básica primaria de la Institución Educativa Nuestra Señora de Fátima del Municipio de Espinal Tolima en Colombia. Los resultados de la investigación fueron positivos, observándose una mejoría en el desempeño de la lectura crítica. De igual forma, se evidenció como la mediación tecnológica en el proceso de enseñanza y aprendizaje contribuyó a la motivación de los estudiantes y al fortalecimiento del proceso de enseñanza y aprendizaje.

Palabras clave: aprendizaje en línea; club virtual; enseñanza; lectura crítica; semiótica.

Clube Virtual: estratégia de ensino e aprendizagem para fortalecer a leitura crítica

Resumo. A implementação de um clube de leitura virtual como estratégia de ensino e aprendizagem surge com o objetivo de fortalecer a leitura crítica. Para apoiar epistemologicamente a proposta, foi estabelecido um marco teórico conceitual. Metodologicamente, propõe-se uma pesquisaação, aplicando o método qualitativo. Neste sentido, elabora-se uma proposta de mudança para a comunidade educacional. A população participante era composta por quarenta (40) estudantes do ensino fundamental (básico) da instituição de ensino Nossa Senhora de Fátima, no município de Espinal Tolima, na Colômbia. Os resultados da pesquisa foram positivos, observando-se uma melhoria no desempenho de leitura crítica. Da mesma forma, evidenciou como a mediação tecnológica no processo de ensino-aprendizagem contribuiu para a motivação dos estudantes, bem como para o fortalecimento do processo de ensino-aprendizagem.

Palavras-chave: aprendizagem on-line; clube virtual; ensino; leitura crítica; semiótica.

Virtual Club: teaching and learning strategy to strengthen critical reading

The implementation of a virtual reading club as a teaching and learning strategy arises with the purpose of strengthening critical reading. In this sense, the theoretical and conceptual framework was founded to support the proposal epistemologically. At the methodological level, research is proposed under the qualitative method with research-action design, in this sense a change proposal is designed for the educational community. The population was made up of forty (40) elementary primary students of the Educational Institution Our Mrs. of Fatima of the Municipality of Espinal Tolima in Colombia. The results of the research were positive, with an improvement in the performance of the critical reading. In the same way, it was evidenced how the technological mediation in the teaching and learning process contributed to the motivation of the students and to the strengthening of the teaching and learning process.

Keywords: online learning; virtual club; teaching; critical reading; semiotics. 


\section{Introducción}

El desarrollo expositivo del artículo se realiza en tres momentos: inicialmente se destacan los aspectos del problema, seguidamente se presenta el marco teórico que orientó la propuesta, a su vez, se aborda el diseño metodológico y finalmente las conclusiones y resultados. En este sentido, con relación al problema que dio origen a la propuesta, se ubica la población objeto de estudio en la Institución Educativa Técnica "Nuestra Señora de Fátima", plantel de carácter oficial con 68 años de historia, el cual cuenta con una población de 720 estudiantes, de los cuales, 40 cursan quinto grado de primaria en el 2018. Los estudiantes oscilan entre los 9 y 12 años de edad, provenientes de familias con un bajo nivel socioeconómico y una visión alejada de la realidad social donde habitan. Se ha evidenciado un bajo desempeño académico en los estudiantes, presentando dificultades en las habilidades relacionadas con la lecto-escritura, entre estas: lectura crítica, análisis de texto, reflexión inferencial de su realidad, análisis gramatical, adicionalmente la disciplina y hábito por la lectura. De igual manera, a las anteriores limitaciones, se le suma la ausencia de estrategias de enseñanza y aprendizaje por parte de los docentes. En consecuencia, de todo lo mencionado anteriormente, se reflejan bajos resultados en las evaluaciones, es así que, en las pruebas denominadas "Martes de prueba de Los Tres Editores S.A.S", los estudiantes obtuvieron un puntaje de 276,89 sobre 500 puntos, con una desviación del 44.58 y un rango promedio de 173.33 , ubicándolos en un nivel de desempeño bajo frente a la Institución.

Por otra parte, en un contexto internacional el programa para la Evaluación Internacional de Alumnos (PISA), para Colombia 2015 y 2018, los resultados en lectura (412 puntos), matemáticas (391) y ciencias (413), se evidencia que los resultados del 2018, son menores que los obtenidos en el 2015 (PISA, 2018); de esta manera emerge un área de trabajo con los estudiantes, teniendo en cuenta que la lectura es fundamental para todas las áreas del conocimiento.

No obstante, el puntaje promedio obtenido en la aplicación 2018 por todos los países participantes de Latinoamérica, al igual que el puntaje promedio de todos los países de la OCDE, mostró disminuciones en los puntajes de las tres áreas (Lectura, Matemáticas y Ciencias) evaluadas entre las aplicaciones de 2015 y 2018.

De otra parte, los datos de Colombia muestran que entre 2006 y 2018 la brecha entre hombres y mujeres ha disminuido en dos de las tres áreas evaluadas. Por ejemplo, en Lectura, en 2006 las mujeres obtuvieron un puntaje 19 puntos más alto que los hombres y en 2018 la diferencia se 
redujo a 10 puntos. De forma similar, en 2006, en Matemáticas los hombres obtuvieron 22 puntos más que las mujeres, mientras que en 2018 la brecha observada es de 20 puntos (MEN, 2018).

Frente a la problemática anteriormente expuesta, surgen diversas inquietudes que originan la búsqueda de soluciones frente a las dificultades evidenciadas en la comunidad educativa. La investigación se orienta desde la siguiente pregunta ¿Cuáles estrategias de enseñanza y aprendizaje pueden incorporarse para el fortalecimiento de la lectura crítica?

De esta manera, en la búsqueda de estrategias para promover la motivación por la lectura, se menciona lo expuesto por Manso (2014), donde se plantea el siguiente interrogante ¿Cómo fomentar y promover el gusto por la lectura en las nuevas generaciones de lectores? El autor indica que se debe partir por el reconocimiento de las necesidades y preferencias de los lectores, tema complejo por la diversidad de estudiantes que se tienen en un grupo. Por otro lado, la tecnología y el auge de los medios audiovisuales han propiciado un cambio en el modelo cultural, pasando de la supremacía de una cultura alfabética, textual e impresa a un modelo construido a base de imágenes audiovisuales (p. 402). Siguiendo a Manso (2014), con su propuesta de aprovechar la relación existente entre tecnología y estrategias de motivación hacia los estudiantes, se propuso el Club Virtual de Lectura (de aquí en adelante CVL), desde una estrategia mediada por las Tecnologías de la Información y la Comunicación (TIC) para promover el interés hacia la lectura e innovar en estrategias de enseñanza y aprendizaje para la práctica docente.

En este sentido, el CVL para los estudiantes significa acercarse al aprendizaje sin la influencia de la calificación y la obligación impuesta por otros, son los encargados de darse sus límites y autoevaluarse. Así, la lectura semiótica de la vida, del cine, de señales y signos, permitirán un aprendizaje vivencial más estimulante y eficaz. También, permitirá la adquisición de una mayor capacidad de observación, discriminación y análisis, convirtiéndolos en personas integrales y autónomas. De esta manera, lograrán una interacción constructiva mediante la incorporación de las TIC en su acercamiento a la experiencia de la lectura.

\section{Referentes teóricos}

La propuesta se sustenta en los referentes entre la mediación de la tecnología mediante un CVL y la experiencia de los estudiantes hacia el acercamiento de la lectura crítica. En este apartado se hace énfasis en la im- 
portancia de saber leer, la incidencia de la lecto-escritura como la interrelación comunicativa, en la cual las habilidades del lenguaje: leer y escribir, tienen un papel simbiótico. En este orden de ideas los referentes teóricos se orientan hacia el reconocimiento de la lectura, la lectura crítica y sus elementos como proceso indispensable en el desarrollo social del ser humano, seguidamente se explicitan los entornos propicios en la lectura (textos impresos y entornos digitales) y las diferentes formas de lectura.

Iniciando la fundamentación teórica se plantean algunos interrogantes ¿Es posible hablar de procesos de producción lingüística separando la lectura de la escritura? ante esto se deben considerar de manera conjunta, en los cuales cada uno pueda complementar el otro. No obstante, la enseñanza y aprendizaje se convierten en actos puramente mecánicos, carentes de sentidos y significados que logren motivar a niños y niñas, que se acercan por vez primera al mundo de la lectura (Velasco, 2009).

Saber leer es de vital importancia, la lectura y la escritura son parte de todas las dimensiones que hacen parte de nuestra integralidad como seres humanos. Evolucionan como un proceso que se da durante toda la vida (Viñas, 2015), leer y escribir son procesos de construcción de sentido que no se agotan en el texto. Leer y escribir van una de la mano de la otra y se aprenden constantemente (Stranges, 2016)

Así, es necesario definir los elementos integrantes de un texto, según Vitale (2020) en su libro El estudio de los signos: Peirce y Saussure, los define como un conjunto finito de construcciones metalingüísticas usadas para definir otras unidades lingüísticas como debe ser la "semántica de los lectores", de esta manera, la semántica cubre el plano intelectual desde la percepción hasta los conceptos abstractos, los cuales son producto de las transacciones y el pensamiento humano.

Por otra parte, la semiótica, permite definir los signos y las formas en que se construye y se transmite el sentido durante la comunicación. El CVL es un espacio para experiencias semióticas discursivas interactivas integrando signos y objetos en su construcción. Es importante destacar que la "primera experiencia lectora" es, definitivamente, crucial. Si la lectura resulta provechosa, todo irá bien, desde este sentido se puede potencializar la incorporación de la estrategia el CVL.

Asumiendo el papel del lector frente a la lectura crítica, debe comprender y tener una visión global del texto, asimilando de manera pragmática, y construyendo ideas relevantes a partir de las ideas principales encontradas 
en el texto. También, debe deducir la información implícita y la posición del autor frente al tema, al evaluar de manera consiente el texto, y tener la visión de otros autores (ICFES, 2017).

En Colombia para las pruebas de Estado sobre la lectura crítica se evalúan tres competencias: (a) el estudiante identifica los contenidos locales que conforman el texto; (b) el estudiante comprende y analiza las partes que estructuran el texto; (c) el estudiante tiene la capacidad para evaluar el texto, analizarlo y dar una opinión sobre el mismo. De acuerdo con lo anterior, es importante generar estrategias que permitan dinamizar el acercamiento del estudiante hacia la lectura.

Por otra parte, un Club de Lectura se plantea como un instrumento para fidelizar lectores apoyado en las herramientas digitales (Moreno et al. , 2017), siempre y cuando, se gestionen y dinamicen de la manera adecuada, para conseguir propuestas teóricas innovadoras en la generación del hábito lector, apoyándose en el concepto de "aprendizaje dialógico" (Valls et al., 2008). Los Clubes de Lectura se vienen trabajando a nivel mundial, consiste en conformar un grupo de personas que realizan actividades en torno a un libro o fragmento propuesto por el líder del club, desarrollando de manera lúdica y pedagógica habilidades de comunicación, escritura y lectura crítica (Aranda y Galindo, 2009).

\subsection{Entornos propicios para la promoción de la lectura}

Los clubes de lectura se constituyen en una de las herramientas más poderosas de promoción lectora, estos se pueden realizar de manera individual y sin un espacio predeterminado, seleccionando un libro para su revisión. Por tanto, son espacios que, a través de la lectura y la conversación permiten la adquisición de diferentes habilidades (Moreno et al., 2017)

\subsection{El lector y su papel en el entorno digital}

Actualmente las mediaciones tecnológicas han transformado las formas de leer, en este contexto de la lectura digital, surgen diversos interrogantes ¿De qué manera nos relacionamos con los nuevos artefactos digitales? ¿Qué incidencia tiene lo multimodal en las nuevas formas de acceder a la lectura? ¿Cuáles son los nuevos tipos de relaciones existentes entre el lector y las nuevas representaciones? son algunas de las preguntas a las que es preciso responder para articular la relación existente entre las nuevas mediaciones tecnológicas y la lectura. El lector digital implica otras habilidades adicionales a las del lector en medios impresos, estas en su gran mayoría se identifican en el abordamiento de la tecnología. 
Así, es diferente la experiencia del lector con los textos impresos, estos son constantes y palpables, físicamente se pueden representar mediante un soporte. Por otra parte, los dispositivos digitales puedan almacenar y desplegar una serie de textos proporcionando fácil uso y portabilidad (Cordón, 2016). Consecuentemente con la experiencia del medio al cual el lector se enfrenta, el lector tiene un interés hacia su objetivo en la lectura, sin embargo, el medio por el cual accede puede permitir que esta experiencia se diferencie de otras.

\subsection{Nuevas formas multimodales de la lectura}

El devenir de las Tecnologías de la Información y la Comunicación (TIC) en los contextos educativos (Silva et al., 2019), ha permitido la evolución hacia nuevas formas multimodales para la lectura, incorporándose la hipertextualidad para albergar múltiples contenidos en línea. A medida que las pantallas están reemplazando al papel como principal sustrato de lectura, la digitalización está influyendo en las actividades de lectura y alfabetización en los diferentes niveles de la educación (Mangen y Weel, 2016).

En este orden de ideas, el presenta trabajo propone un Club Virtual, como estrategia apoyada en la tecnología superando la limitación de tiempos y espacios de la población objeto de estudio, y, por otra parte, como una alternativa motivadora para los estudiantes en su experiencia de aproximación hacia la lectura crítica. Asimismo, fue necesario la incorporación de herramientas tecnológicas para su construcción, se identificó la plataforma Edmodo para implementar el CVL, la selección se orientó desde sus características como una plataforma innovadora, fácil, accesible y gratuita (EDMODO, 2019).

Edmodo es una plataforma educativa que presenta una manera interactiva de aprender, a la cual puede ingresar bajo tres roles, el docente, el cual es el encargado de crear el grupo, o clase y plantear las actividades, los estudiantes pueden acceder en segunda instancia si tiene el código para ingresar al curso, y el de invitado, para familiares de los estudiantes. Adicionalmente cuenta con una serie de ventajas para los profesores y alumnos (EDUCACIÓN 3.0, 2018), entre estas (a) Gamificación: se integra a través de las insignias, que son creadas por el profesor y otorgadas a los alumnos (b) Seguridad: es una red segura para padres, alumnos y profesores, los datos se muestran en la plataforma de manera confidencial. La plataforma educativa Edmodo, también es una red social, en la que los estudiantes y profesores pueden interactuar de manera positiva, frente a su avance en el proceso de enseñanza y aprendizaje. 


\section{Metodología}

Se desarrolla una metodología de enfoque cualitativo y un diseño de investigación acción, esta es considerada como una metodología que permite dar respuestas a los diferentes problemas en contextos educativos, siendo los estudiantes el centro de la investigación, dando la posibilidad a cada estudiante para que pueda tener herramientas para construir su propio conocimiento (Sequera, 2014).

De acuerdo con Martí (2012) se presentan las fases de la investigación acción (IA) así: (a) Preparatoria, (b) Trabajo de campo, (c) Analítica y (d) Informativa.

- Fase preparatoria: se materializa la idea, y la relevancia del problema "la fase preparatoria está constituida en dos etapas: reflexiva y diseño".

- Fase de trabajo de campo: se planifica de manera teórica y práctica las actividades a desarrollar y se fundamenta la ejecución con ayuda de expertos en el tema. Se diseñan las estrategias y se implementa la propuesta pedagógica.

- Fase analítica: después de aplicar la implementación de la estrategia pedagógica, se hace necesario verificar la hipótesis, esto mediante la aplicación de instrumentos de validación, que serán descritos más adelante.

- Fase informativa: se analizan los resultados de la aplicación del instrumento, después de la aplicación de la propuesta pedagógica. Se tienen el cuestionario oral y grabación en video para evaluar expresiones y comentarios puntuales de los estudiantes.

Sobre los instrumentos de recolección de información son necesarios para este proceso, por ello hay que construirlos desde el enfoque cualitativo, entre estos se tienen: la prueba diagnóstica, prueba de lectura crítica, instrumento de grupo focal dirigido a estudiantes, entrevista de validación de la estrategia, diario de campo, este último se aplica sobre la observación de los logros del encuentro, consignando las dificultades que se presentaron los estudiantes inherentes a la actividad del CVL. Para la muestra de la investigación se han tomado estudiantes del grado décimo, el cual está compuesto por 40 estudiantes, la muestra utilizada es no probabilística. 


\subsection{Secuencia didáctica}

Luego del análisis de la prueba diagnóstica, se establecen los parámetros para diseñar el $\mathrm{CVL}$, partiendo de las dificultades evidenciadas en el proceso de comprensión textual e intertextual de los estudiantes de grado décimo. De esta manera, se plantea un plan de trabajo, donde se diseñan actividades amenas, motivadoras y constructivas, encaminadas a formar lectores críticos y con interés hacia la lectura. Así, las actividades están diseñadas para motivar a los estudiantes a leer de manera crítica, fortaleciendo su competencia lecto-escritora.

De esta manera, a través del uso de la plataforma Edmodo mediante un $\mathrm{CVL}$, se complementarán actividades dentro del aula. Además de trabajar significativamente desde el contexto con la obra literaria la vendedora de claveles de Andrés Elías Flórez Brum (Flórez, 2001), que trasluce la realidad, de la mayoría de estudiantes. Por tanto, las sesiones a trabajar en esta propuesta pedagógica se encaminan a desarrollar los propósitos presentados en esta investigación, cada una de las sesiones tiene una duración de 6 horas, distribuidas en diferentes clases, las cuales se presentan en la Tabla 1.

Tabla 1. Sesiones del club virtual de lectura

\begin{tabular}{ll}
\hline Sesión 1 & Inauguración del club de ledtura virtual Matices \\
Sesión 2 & Ahora soy miembro del club \\
Sesión 3 & Debatiendo lo que pienso \\
Sesión 4 & Atrévete a responder \\
Sesión 5 & Concurso: poniendo a volar mi imaginación \\
Sesión 6 & Construyendo una brújula \\
Sesión 7 & El rol del periodista \\
Sesión 8 & Carta a la vendedora de claveles \\
Sesión 9 & Disco foro: Aprender a quererte
\end{tabular}

Fuente: Elaboración propia.

A continuación se presenta la planeación de una de las sesiones, para este caso la sesión 6 correspondiente con Construyendo una brújula (ver Tabla 2), las cuales contienen: tema, tiempo, estándar, derechos básicos de aprendizaje (DBA), objetivo de aprendizaje y descripción general de la actividad, y el trabajo a realizar en la plataforma Edmodo. Es de aclarar que las demás sesiones propuestas siguen la misma planeación, que la evidenciada para la fase 6 . 
Tabla 2. Planeación de la sesión 6 construyendo una brújula

Tema Construcción de un esquema mental, luego de un proceso meta verbal, semántico discursivo

Tiempo 6 horas

Estándar Elaboro hipótesis de interpretación atendiendo a la intención comunicativa y al sentido global del texto que leo

Diseño un esquema de interpretación, teniendo en cuenta el tipo de texto, tema, interlocutor e intención comunicativa

DBA Tiene en cuenta la progresión temática del texto que se propone a producir y reconoce como la información nueva-tema-debe articularse con la información-tema

Objetivo de Construir un mapa mental, haciendo uso de la herramienta de aprendizaje aprendizaje Cmaptooll

Descripción 1. Descargar el mapa mental en la computadora

general de la actividad

2. Completar el mapa mental de acuerdo a la lectura que realizaste de la obra literaria la vendedora de claveles

3. Vuelve a cargar tu trabajo en esta asigación

4. Visualiza los trabajos de tus compañeros y escoge una idea relevante que te haya llamado la atención

5. Puedes hacer grupo de dos personas, para esta actividad

Fuente: Elaboración propia.

En la Figura 1 se detallan las actividades dispuestas en la plataforma virtual donde se encuentra disponible el CVL, se puede evidenciar la sesión 5 Poniendo a volar mi imaginación, donde se presentan las actividades que se adelantarán en la plataforma por parte de los estudiantes.

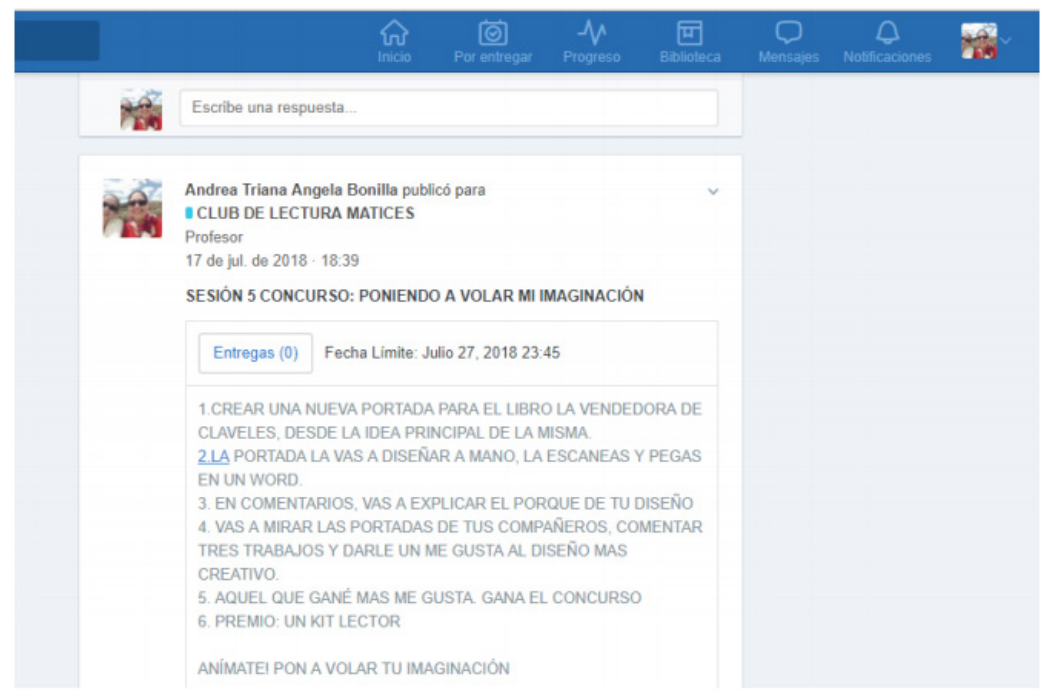

Figura 1. Sesión 5 dispuesta en el Club Virtual de Lectura Matices 


\subsection{Componente tecnológico}

En cada sesión se hace uso de la plataforma Edmodo, abordando las diferentes formas de lectura e interpretación crítica; una de las ventajas es su diseño similar a la red social Facebook, haciéndola innovadora y fácil de usar, se accede con usuario y clave. En esta plataforma (ver Figura 2) se puede compartir links, crear debates, visualizar los trabajos de sus compañeros, crear evaluaciones. Otro valor agregado consiste en que puede descargarse por Play Store, y manejarse por medio del dispositivo celular.

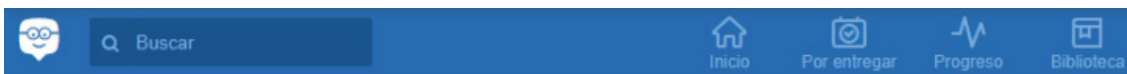

Andrea Triana Angela Bonilla publicó para

- CLUB DE LECTURA MATICES

Profesor

21 de jun. de $2018 \cdot 20: 29$

SESIÓN 2

HOLA CHICOS

BIENVENIDOS A ESA MARAVILLOSA AVENTURA

LUEGO DE COMPARTIR JUNTOS, CON ROBERTO, JOSE WILINTON Y

LLORAR CON LA VENDEDORA DE ROSAS, LA PERDIDA DE SU HIJA ADRIANA ANDREA.

VIAJEMOS JUNTOS POR LA LITERATURA, QUE NOS LLEVA A VIVIR VIDAS QUE NO HEMOS VIVIDO

NUEVAMENTE BIENVENIDOS A EST Más.

Figura 2. Club Virtual de Lectura Matices

\subsection{Implementación}

A continuación se presenta la planeación y estrategias con las que se abordó el CVL, desde una propuesta didáctica para el mejoramiento del proceso lector, la motivación hacia la lectura y las pautas para despertar en los estudiantes el interés por una crítica constructiva, reflexiva y social, frente a los textos que leen y vivencian a diario.

\subsection{Metodología utilizada en cada sesión de clase}

La metodología a utilizar es lúdico pedagógica interactiva, que se adapta a las situaciones del contexto de cada estudiante, donde interactúa con una obra literaria que se adapta a su medio, de manera constructiva, lo que le permite despertar su interés por la literatura y la interactividad que lo lleva a bordar la lectura del libro desde otros ámbitos, creando espacios donde puede juzgar, apoyar, y expresar lo que siente referente a la lectura. Fortaleciendo sus competencias lecto escriturales y comunicativas, necesarias 
para abordar su contexto de manera crítica, generando cambios desde su interacción con el mundo que le rodea, planteando así, soluciones reales a sus problemas cotidianos.

Primero. Uso de la plataforma Edmodo: para el desarrollo de la propuesta que pretende motivar el interés del estudiante por la lectura crítica, como un medio interactivo, que lo traslada del aula de clase, a un espacio conocido por él, y en el cual le agrada interactuar, cuyas actividades se vuelven flexibles e integrales y las cuales puede compartir con sus compañeros, y complementar lo aprendido en el aula de clase.

\section{Segundo. Métodos didácticos:}

- El método global, en el cual se hace una exploración general antes de entrar a trabajar en detalles específicos de la lectura; una ruptura de la lectura lineal del texto, ya que se vuelve sobre distintos capítulos, para la contextualización del mismo; se aborda el texto de lo general a lo particular, de lo conocido a lo desconocido, anticipando el sentido, pre-suponiendo, sirviéndose del contexto para descubrir palabras desconocidas.

- El método semiótico-discursivo que tiene en cuenta el texto y el contexto natural, el contacto con el entorno y su interpretación. Llegando a la Metacognición haciendo relaciones, asociaciones, creaciones, para interpretar, asimilar, inferir las significaciones, enriqueciendo el texto.

- El método semántico-discursivo: haciendo interpretación de significados, polisemia, antonimia, sinonimia y otros.

- El método de representación y creación artística: donde los alumnos se apropian de los textos y son recreados por ellos mismos.

Tercero. Rol del estudiante: asume un rol participativo y de su construcción de su propio conocimiento, adentrándose a la lectura crítica de manera idónea y sin sentirse obligado por una responsabilidad académica, siendo autónomo de su tiempo.

Cuarto. Rol del docente: facilitador del conocimiento y motivador del proceso lector de los educandos, aportando al crecimiento integral de los estudiantes. Es encargado de la generación de espacios de debate, proposiciones, y diálogos, donde el estudiante es el protagonista.

Quinta. Selección de la obra: la obra literaria se lee desde el aula y se trabaja de manera interactiva proporcionando pautas para una lectura crítica contextualizada, a partir de actividades que se desarrollan en la plataforma.

Sexta. Inicio de la sesión: se inicia con la motivación preliminar realizada en el aula, la cual involucra una actividad meta verbal, este es el punto de partida donde el estudiante emprende su proceso autónomo como miembro 
del CVL. Seguidamente se invita a los estudiantes a compartir sus trabajos a través de la plataforma, leer el de sus pares y comentarlos. El estudiante debe evaluar la percepción que se tiene frente a la manera de expresión, estilo, cohesión y coherencia, al igual que el punto de vista y opinión de los demás. Antes de ir a la plataforma educativa, se ha leído el libro con los estudiantes y realizado un proceso de motivación y sensibilización.

La interacción con la plataforma virtual, se enriquece a través del uso de otras herramientas virtuales entre las que resaltamos: CmapTools, Cava, Office. Al inicio de cada sesión se hace una retroalimentación permanente, que permite mantener una motivación constante en el estudiante y se incentiva la comunicación virtual por la plataforma, con el docente y sus pares.

Séptima. La evaluación: utilizada durante todo el proceso, evaluando de manera integral el proceso de avance, en la Tabla 3 se evidencia las fases previstas para la evaluación de los estudiantes.

Tabla 3. Procesos de evaluación

Proceso de evaluación

Autoevaluación Ejercicio individual y autónomo en donde el estudiante toma en cuenta sus falencias y fortalezas frente al proceso educativo

Coevaluación En esta etapa se cuenta con la opinión de otro, que posibilita ampliar su análisis personal y fortalece los procesos académicos

Heteroevaluación En esta fase hay un acercamiento entre el docente y el estudiante donde se consolida la visión frente al desarrollo, y del estudiante sus fortalezas y debilidades en el proceso académico

Fuente: Elaboración propia.

\section{Análisis y resultados}

Para el análisis de los resultados se presenta una comparación con la realidad comunicativa de los estudiantes antes de abordar la propuesta, en la cual se evidencia un mejoramiento de la lectura en nivel crítico, así como en la motivación hacia una lectura amena y critica, ya que ellos mismos tomaron la iniciativa y autonomía en el desarrollo de las sesiones del CVL, en la Tabla 4 se reflejan los resultados.

Después se presentan los resultados de la prueba final que evalúa las competencias expuestas anteriormente. La prueba muestra las habilidades en lectura crítica de los estudiantes. 
Tabla 4. Diferencias entre la fase diagnóstica y la fase de cierre

Fase diagnóstica

Competencias que requieren mejoras

Se les dificulta realizar juicios de valor sobre los textos que leen, dejando de un lado el planteamiento de hipótesis frente a los interrogantes del mismo texto

No hay una apropiación del texto, no se hace esa lectura intertual, adaptándolo o visualizándolo en su realidad
Fase de cierre

Competencias y habilidades adquiridas en la aplicación y apropiación de la propuesta pedagógica

Elaboran hipótesis y las interpretan, dando respuesta a la intención comunicativa de un texto

Contextualizan el texto, de manera vivencial, haciendo una lectura intertextual del mismo

Identifican los diversos tipos de textos y su intención comunicativa

Leen y entienden los textos desde la dimensión ética, filosófica

Analizan la forma y el fondo de los textos que leen

Fuente: elaboración propia.

Al aplicarse la prueba de lectura crítica se presentó un texto sobre el cual se harían preguntas relativas a la comprensión lectora, se pudo obtener como resultado en los estudiantes un $62 \%$ que acertó de manera correcta, mientras que el $38 \%$ respondió a la formulación de manera incorrecta, siendo un porcentaje favorable hacia la implementación del CVL.

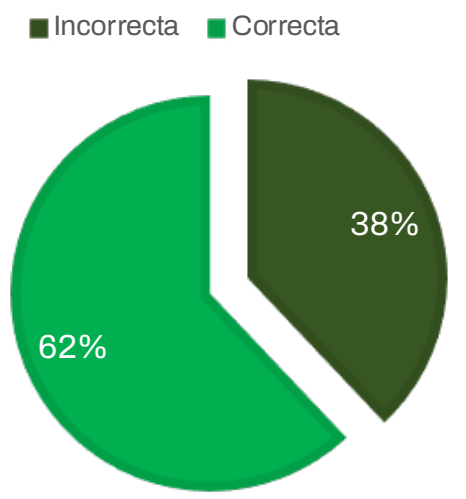

Figura 3. Resultados de las pruebas sobre Lectura Crítica

Por otra parte, el análisis del instrumento utilizado en el grupo focal para medir el impacto de la estrategia pedagógica de un CVL en la plataforma Edmodo sobre lectura crítica, evidenció comentarios positivos por parte de los estudiantes. 
Para finalizar es importante mostrar los niveles de desempeño de los estudiantes (ver Figura 4), resultados obtenidos mediante la aplicación del instrumento para la validación de la lectura crítica. En este sentido, los estudiantes en promedio para el nivel básico obtuvieron un $62 \%$; en el nivel alto $20 \%$; nivel bajo $10 \%$ y finalmente en un nivel superior $8 \%$. De esta manera, la mayoría de los estudiantes se encuentran ubicados en un nivel alto, básico y superior.

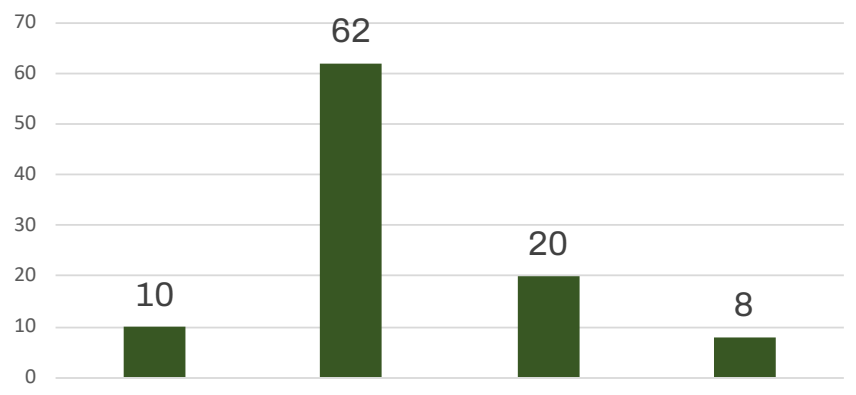

Figura 4. Desempeño final prueba de lectura crítica

Por tanto, se puede apreciar que la implementación de la estrategia didáctica del CVL, permitió en los estudiantes superar la prueba de desempeño final frente a la de desempeño inicial, los anteriores resultados se aprecian en la Figura 5.

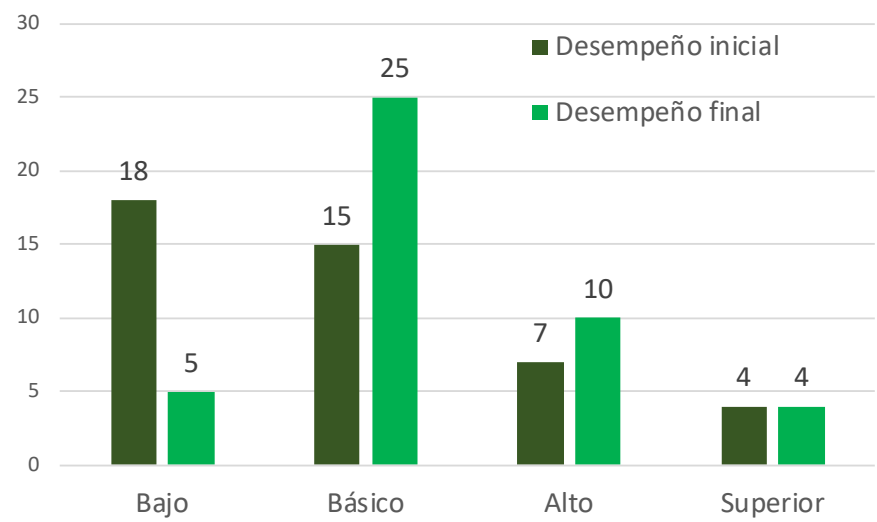

Figura 5. Comparación desempeño inicial frente al desempeño final de la prueba sobre lectura crítica 


\section{Conclusiones}

La implementación de un CVL para el fortalecimiento de la lectura crítica, se constituyó en una estrategia didáctica innovadora para el proceso de enseñanza y aprendizaje, consolidando resultados positivos. Se destaca el avance en cuanto a la construcción de la interacción entre pares para construir y analizar textos, reconocimiento de contenidos, establecer nexos intertextuales y por tanto comprender el valor del lenguaje para la construcción de conocimiento

EI CVL se diseñó un conjunto de estrategias pedagógicas en línea, a través de la plataforma Edmodo, dirigido especialmente para los estudiantes de décimo grado de la Institución Educativa Nuestra Señora de Fátima del Municipio de El Espinal Tolima. En general, la prueba inicial permitió identificar las dificultades iniciales de los estudiantes, las cuales fueron mitigadas por medio de la implementación de la propuesta pedagógica. De esta manera, se evidencia como las plataformas virtuales tienen funciones particulares en los procesos educativos (Sajoza, 2018), y cómo las TIC pueden ayudar en la solución de determinada situación (Bossolasco et al., 2018), por consiguiente la propuesta de un CVL, evidenció una estrategia innovadora para la enseñanza y aprendizaje de la Lectura Crítica. Es de vital importancia el papel que desempeña el docente, en algunas ocasiones las TIC a pesar de estar en las escuelas no son aprovechadas al máximo (Silva y Montañez, 2019). Sin lugar a dudas, se evidencia en esta propuesta como la utilización de las TIC permitió convertir una plataforma virtual en un CVL desde el trabajo en un aula presencial, (Silva, Mendoza y Girado, 2018).

Por otra parte, se evidencia la construcción del pensamiento analítico y crítico que adquirieron los estudiantes, adicionalmente los resultados contribuyeron en el afianzamiento de su autoestima, a la valoración y respeto por el otro, al replanteamiento de metas individuales y grupales, en donde la tecnología se convierte en un medio para alcanzar el fin. Este club se ofrece como un "grupo piloto" dentro de la institución, mediante el cual se van a aplicar una serie de estrategias metodológicas que contribuirán al mejoramiento pedagógico y del currículo, aprovechando el recurso humano y tecnológico existente.

De igual manera, se destaca la importancia en la generación del habito de la lectura en edades tempranas, mediante la incorporación de un CVL. En este sentido la mediación tecnológica y la construcción colectiva acompañada de un par lector, permitió motivar al estudiante, en cuanto al apoyo con su grupo de lectura, y la motivación intrínseca por el logro propuesto en cada encuentro de lectura. 
Asimismo, la representación de diferentes mediaciones tecnológicas para el mejoramiento en el fomento de la lectura, se constituye en un reto para los educadores entendiendo que la tecnología no puede sustituir el apoyo docente en el acto educativo. No obstante, se destaca que la tecnología cumple un papel de motivación en los estudiantes, al abordar los contenidos de manera digital. Finalmente, los estudiantes de grado décimo serán los grandes beneficiados en el ejercicio de la actividad lectora, al igual que un aporte para los futuros estudiantes de la Institución y una propuesta para docentes que la quieran implementar. También, se vislumbra como una propuesta que puede ser adoptada en diferentes instituciones educativas con el fin de generar escenarios innovadores para incentivar la lectura en los estudiantes.

\section{Referencias}

Aranda, J. y Galindo, B. (2009). Leer y conversar. Una introducción a los clubs de lectura. Gijón: Trea.

Bossolasco, M. L., Casanova, B., Enrico, E., Dos Santos, D. y Enrico, R. (2018). Perfiles de apropiación de TIC y desempeño académico en estudiantes universitarios: Un estudio de caso utilizando analítica del aprendizaje. Virtualidad, Educación y Ciencia, VEC, 1-18.

Cordón, J. A. (2016). La investigación sobre lectura en el entorno digital. MEl: Métodos de información, 7(13), 247-268.

EDMODO (2019). Plataforma Edmodo. Recuperado de https://www.edmodo.com/

EDUCACIÓN 3.0 ( 2018). Líder informatico en innovación educativa. Recuperado de https:// bit.ly/34S16pRml

Flórez, A. (2001). La vendedora de claveles. Bogotá: Educar.

ICFES (2017). Escala de desempeño según el MEN, en el Decreto 1290 de 2009. Instituto Colombiano para el Fomento de la Educación Superior. Recuperado de https://bit. ly/3ryRJoJ

Los Tres Editores SAS (2017). Martes de prueba. Recuperado de http://www.lostreseditores.com

Manso, R. (2014). Bibliotecas, fomento de la lectura y redes sociales: convirtamos amigos en lectores. El profesional de la información, 21(1), 401-405.

Mangen, A. y Weel, A. (2016). The evolution of reading in the age of digitisation: an integrative framework for reading research. Literacy, 50(3), 116-124.

Martí, J. (2012). La investigación Acción Participativa: Estructuras y Fases. Recuperado de https://bit.ly/3aTfZfk

MEN (2018). Pruebas PISA. Ministerio de Educación Nacional. Recuperado de https://bit. Iy/3aRFUnQ 
Moreno, M., García-Rodríguez y Gómez-Díaz, R. (2017). Conversando en la nube: cómo organizar un club de lectura. Revista General de Información y Documentación, 27(1), 117199. https://doi.org/10.5209/RGID.56566

PISA (2018). Programa para la Evaluación Internacional de Alumnos. Colombia. Recuperado de https://bit.ly/3n36flm

Sajoza, V. (2018). Algunas miradas sobre las TIC y la inmediatez en la educación. Virtualidad, Educación y Ciencia.VEC, 9(17),1-7.

Sequera, M. (2014). Investigación acción: un método de investigación educativa para la sociedad actual. Revista Arjé, 223-229.

Silva, A. y Montañez, L. F. (2019). Aprendizaje psicomotriz en el área de Educación Física, Recreación y Deportes mediado por el uso de software educativo. Retos, 36, 302-309.

Silva, A., Mendoza, J. y Girado, A. (2018). Prevención del consumo de sustancias psicoactivas. Un aporte desde la neurociencia y el aprendizaje basado en proyectos ABP. Revista Iberoamericana de Educación, 78(1), 107-126. https://doi.org/10.35362/ rie7813214.

Silva, A., Quirós, S., Sandoval, M. y Pacheco, D. (2019). Del cerebro al aula: Conceptos claves desde la Neurociencia y su aporte en la educación. En E. Serna, Revolución en la Formación y la Capacitación para el Siglo XXI, Volumen 1 (pág. 504). Medellín: Instituto Antioqueño de Investigación

Stranges, A. (2016). Inclusión educativa: la importancia de saber leer y escribir. Letras, 31-35.

Valls, R., Soler, M. y Flecha, R. (2008). Lectura dialógica: interacciones que mejoran y aceleran la lectura. Revista Iberoamericana de Educación, 46, 71-87.

Velasco, A. (2009). La lectura y la escritura como procesos transversales en la escuela Experiencias innovadoras en Bogotá. Bogotá: Secretaría de Educación.

Viñas, R. (2015). Tesis Doctoral "Ser joven, leer y escribir en la universidad". La Plata: Facultad de Periodismo y Comunicación Social.

Vitale, A. (2020). El estudio de los Signos: Peirce y Saussure. Buenos Aires: Eudeba. 